\title{
LINEAR PROGRAMMING WITH PYTHON AND PULP
}

\author{
Kanishka Parganiha \\ Department of Mechanical Engineering \\ Medicaps Institute of Technology and Management, Indore, India
}

\begin{abstract}
Linear Programming is a family of mathematical programming that is concerned with the allocation of scarce or limited resources i.e. raw materials, partially finished products, labour, investment capital or time to several competing activities on the basis of given optimality. It is employed in operation research for the purpose of optimization of linear functions subject to linear equality and inequality constraint. An optimal allocation may be one that maximizes some measure of benefit or utility, such as profit, or minimizes some measure of cost. The technique of linear programming is used in wide range of applications, including agriculture, industry, transportation, economic, health system, social science, and military.

In this paper we will solve a linear programming problem using python language. As an analyst, we try to find a good optimal solution for the decision maker to make a final decision. Our attempt is to give the mathematical description of the product-mix optimization problem. The objective of this paper is to find the best mix that maximizes profit. To obtain the solution to this Linear program, we have to write a short program in Python to call PuLP's modeling functions, which will then call a solver. Then we will obtain the graph using python commands, which will give the shaded area enclosed by the constraints called feasible region, which is the set of points satisfying all the constraints. To find the optimal solution we look at the lines of equal profits to find the corner of the feasible region which yield the highest profit. This corner can be found out at the farthest line of equal profit which still touches the feasible region.
\end{abstract}

Keywords: Constraints, Feasible region, GLPK solver, Objective function, Optimization, and PuLP.

Cite this Article: Kanishka Parganiha, Linear Programming with Python and PuLP, International Journal of Industrial Engineering Research and Development, 9(3), 2018, pp. 1-8. https://iaeme.com/Home/issue/IJIERD?Volume=9\&Issue=3

\section{INTRODUCTION}

Linear programming [1] was developed during World War II, when a system with which to maximize the efficiency of resources was of utmost importance and to lay out a path of detailed decisions to take in order to "best" achieve its goals when faced with practical situations of great complexity. Although many business organization see linear programming 
as a new science or recent development in mathematical history, there is nothing new about maximization of profit in any business organization in a production company or manufacturing company. Today linear programming is a standard tool that has saved many thousand or millions of dollar for most companies or businesses. So mathematically linear programming is the technique used to maximize or minimize a function. The idea is to optimize a complex function by best representing them with linear relationships. In simpler terms, we try to optimize (to maximize or minimize) a function denoted in linear terms and bounded by linear constraints. The mathematical function which must be optimised is called an objective function. The objective function and the constraints placed upon the problem must be deterministic and able to be expressed in linear form.

So as we know linear programming is widely used for allocating resources to achieve an objective. Here in this paper the product-mix problem is illustrated as a prototype example [3] refers to manufacturing system which may occur in real life applications is being solved through PYTHON. PYTHON is a general-purpose interpreted, interactive, object- oriented, and high level programming language. In this,we will use python to call PuLP which will then call the solver to solve the linear programming inequalities. Pulp stands for "Python Linear Programming". PuLP is a LP modeler and a free open source software written in python. It is used to describe optimization problems as mathematical problems. PuLP can then call any of numerous external LP (Linear Programming) solver like CBC, GLPK, CPLEX, Gurobi to solve the model display the solution and then use 'NumPy' and 'Matplotlib' library to display feasible region in graphical form.

\section{THE OPTIMISATION PROCESS}

Solving an optimisation problem [2] in general can be broken down into five general steps

- Getting the description of the problem

- Formulating the mathematical problem

- Solving the mathematical program

- Performing the post-optimal analysis

- Presenting the solution and analysis

The modeling starts with a well-defined model description, then uses mathematics to formulate a mathematical program. Next, the modeler enters the mathematical program into some solver software, e.g., Excel and solves the model. Finally, the solution is translated into a decision in terms of the original model description.

Using python gives you a "shortcut" through the modeling process. By formulating the mathematical program in Python you have already put it into a form that can be used easily by PuLP the modeller to call many solver, e.g. CPLEX, COIN, Gurobi, GLPK so you don't need to enter the mathematical program into solver software. 


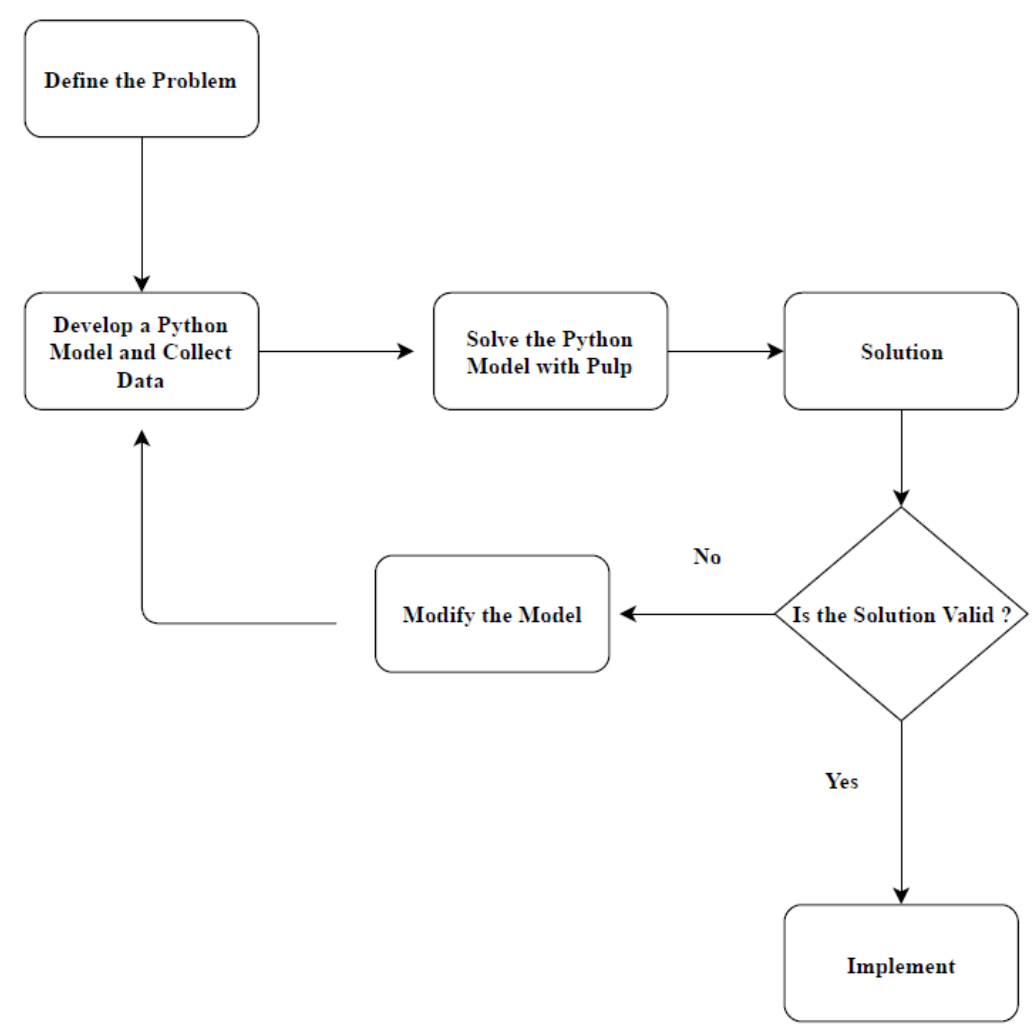

Figure 1 Operation Research Methodology Diagram

The process shown above is known as Operation Research Methodology Diagram. As clearly shown above there are "feedback loops" within this process. For example, after formulating and solving an optimization problem, you will often want to consider the validity of your solution. If your solution is invalid you may need to alter or update your formulation to incorporate your new understanding of the actual problem. So python gives you all this advantages while solving an optimization problem.

\section{PROBLEM STATEMENT}

A farmer has recently acquired an 110 hectares piece of land. He had decided to grow Wheat and barley on that land. Due to the quality of the sun and the region's excellent climate, the entire production of wheat and Barley can be sold. He wants to know how to plant each variety in the 110 hectares, given the cost, net profits and labor requirements according to the data shown below:

Table 1 Cost, net profits and labour requirements

\begin{tabular}{|l|c|c|c|}
\hline \multicolumn{1}{|c|}{ Variety } & Cost(Price/Hectare) & Profit(Price/Hectare) & $\begin{array}{c}\text { Man- } \\
\text { days/Hectares }\end{array}$ \\
\hline Wheat & 100 & 50 & 10 \\
\hline Barley & 200 & 120 & 30 \\
\hline
\end{tabular}

The farmer has a budget of US\$10,000 and a availability of 1,200 man-days during the planning horizon. Find the optimal solution and the optimal value [3]. 


\section{FORMULATION OF LINEAR PROGRAMMING MODEL}

Let the total area for growing Wheat $=\mathrm{X}$

Let the total area for growing Barley $=\mathrm{Y}$

Now, the total profit is represented by $\mathrm{Z}$

Objective Function: Max $Z=50 X+120 Y$

which means we have to maximise $\mathrm{Z}$.

Constraints: $100 \mathrm{X}+200 \mathrm{Y} \leq 10,000$

$$
\begin{aligned}
& 10 X+30 Y \leq 1200 \\
& X+Y \leq 110 \\
& X \geq 0 \\
& Y \geq 0
\end{aligned}
$$

\section{SOLUTION USING PYTHON}

To run program on python 3.7, we will be using Jupyter Notebook which is an open-source web application that supports over 40 programming language including python and allows us to create and share documents that contain live code, equations, visualizations and narrative text.

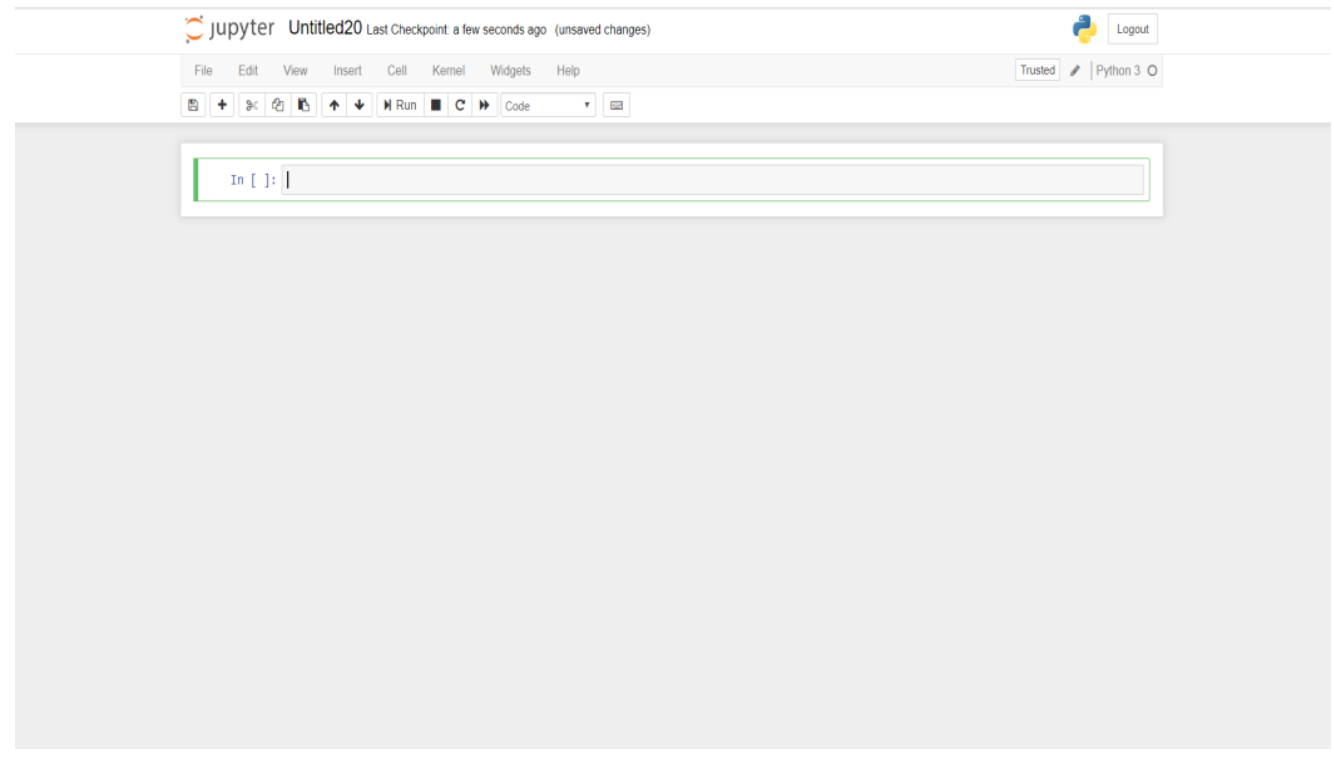

Figure 2 Jupyter Notebook

To start our program in python, we will first import PuLP's modeler function for use in our code:

In [1]: import pulp

Figure 3 Importing PuLP library

A variable "my_lp_problem" is created using the "LpProblem" function. It has two parameters, the first being the arbitrary name of this problem and the second parameter being "LpMaximize" as we have to maximize the objective function $\mathrm{Z}$. 


$$
\text { my_lp_problem = pulp.LpProblem("My LP Problem", pulp.LpMaximize) }
$$

Figure 4 Identifying objective function variable

Now we will create the problem variables ' $\mathrm{X}$ ' and ' $\mathrm{Y}$ ' using 'LpVariable' class. It has four parameters, the first is the arbitrary name of the variable, the second is the lower bound on this

$$
\begin{aligned}
& x=\text { pulp. LpVariable(' } x \text { ', lowBound }=0, \text { cat='Continuous') } \\
& y=\text { pulp.LpVariable(' } y \text { ', lowBound }=0, \text { cat='Continuous') }
\end{aligned}
$$

Figure 5 Defining decision variables

variable, the third is the upper bound, and the fourth is essentially the type of data (discrete or continuous), with the default as 'LpContinuous'.

The variable "my_lp_problem" will now collect problem data with the '+=' operator. The objective function is logically entered first, with an important comma ',' at the end of the statement and a short string explaining the objective function:

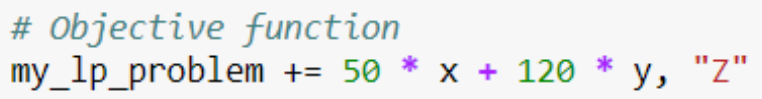

Figure 6 Defining objective function

The constraints are now entered using ' $+=$ ' operator again, since we are adding more data to the 'my_lp_problem' variable.

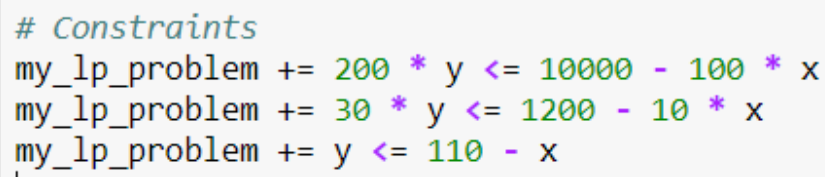

Figure 7 Defining constraints

Now, we have now constructed our problem and can have a look at it. We can do it by type our variable name 'my_lp_problem' and then hit run.

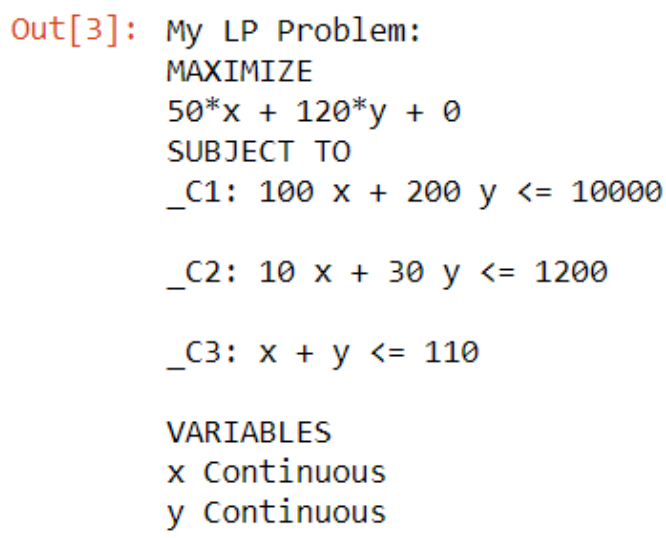

Figure 8 Output of the problem statement as per our input 
Now, the linear programming is solved using the 'solve()' command and the result of the solver call can be displayed to output to us. Firstly, we request the status of the solution, which can be one of "Not Solved", "Infeasible", "Unbounded", "Undefined", "Optimal".

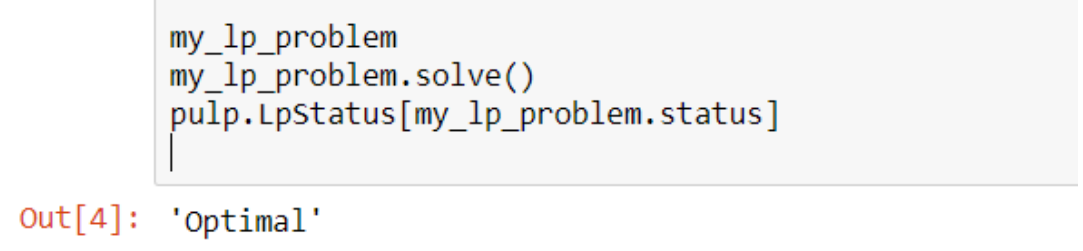

Figure 9 Status of the solution

Since it shows 'Optimal', that means we can proceed with it. The variables and their optimum values can now be printed to the screen. For this we will use 'for' loop as it makes 'variable' cycle through all problem variable name. then it prints each variable name, followed by an equal sign, followed by its optimum value. 'name' and 'varValue' are properties of the object variable.

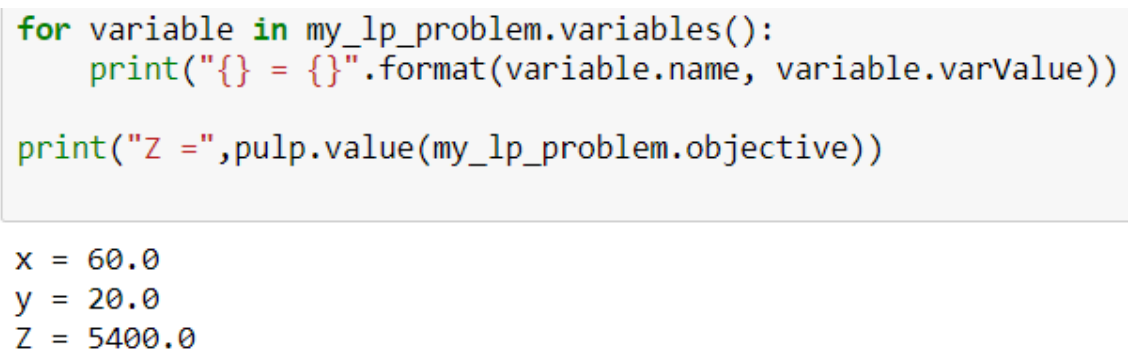

Figure 10 Final solution

By running the program, the optimised objective function ' $\mathrm{Z}$ ' is printed to the screen along with the value of both ' $\mathrm{X}$ ' and ' $\mathrm{Y}$ '. In this way we obtain the maximum net profit for the farmer's product as ' 5400 '.

\section{GRAPHICAL REPRESENTATION OF FEASIBLE REGION USING PYTHON}

For graphical representation of the feasible region we will use 'NumPy' and 'Matplotlib' libraries of python. NumPy [4] is a module for python which stands for "Numeric Python" and its main function is to enriches the programming language Python with powerful data structures, implementing multi-dimensional arrays and matrices. Whereas 'Matplotlib' [5] is a python library used to create $2 \mathrm{D}$ graphs and plots by using python scripts. It supports variety of graphs and plots namely - histograms, bar charts, power spectra, error charts etc. Matplotlib along with NumPy is used to provide an environment that is an effective open source alternative for 'MATLAB'.

Here is the code for the graphical representation of the feasible region are as follows: \#Importing libraries of NumPy and Matplotlib

import numpy as np import matplotlib.pyplot as plt

$\%$ matplotlib inline $\mathrm{x}=$ np.linspace $(0,600,10000)$ 
\#Constraints

$\mathrm{y} 1=(100-\mathrm{x}) / 2$

$\mathrm{y} 2=(120-\mathrm{x}) / 3$

$\mathrm{y} 3=110-\mathrm{x}$

\# Make plot

plt.plot(x, y1, label=r'\$100x+200y\leq10000\$')

plt.plot(x, y2, label=r'\$10x+30ylleq1200\$')

plt.plot(x, y3, label=r'\$x+ylleq110\$')

plt.xlim((0, 200))

plt.ylim((0, 200))

\#Labelling $\mathrm{X}$ and $\mathrm{Y}$ axis

plt.xlabel $\left(r^{\prime} \$ x \$ '\right)$

plt.ylabel(r'\$y\$')

\# Fill feasible region

y5 = np.minimum $(y 2, y 1)$

plt.fill_between(x, y5, color='pink',alpha=0.5)

plt.legend(bbox_to_anchor $=(1.5,1)$, loc $=5$, borderaxespad=0.)

After running the above program we get the desired graphs of the equations. The pink shaded region represents the feasible region i.e. the optimal feasible solution is achieved at the point of intersection of equations $100 \mathrm{X}+200 \mathrm{Y} \leq 10,000$ and $10 \mathrm{X}+30 \mathrm{Y} \leq 1200$ intersects gives us the optimal solution.

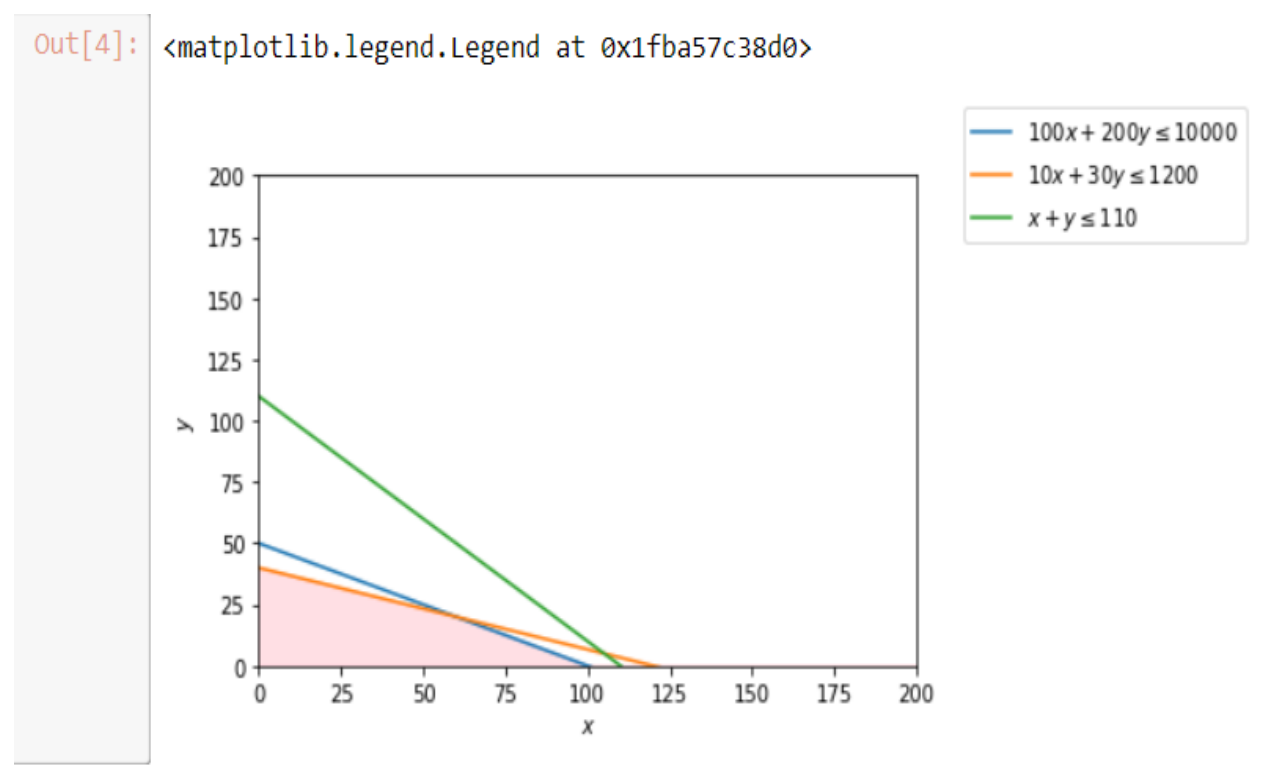

Figure 11 Output of 'Feasible Region' 


\section{CONCLUSIONS}

The Objective of the research work in solving a two variable linear program and getting an optimal solution along with feasible region is achieved. The final result as derived using PuLP are as follows: The value obtained are $\mathrm{X}=60, \mathrm{Y}=20$ and $\mathrm{Z}=5400$ which means that to produce maximum profit the farmer should produce Wheat and Barley in 60 and 20 hectares of land respectively and the maximum profit of the company will gain $5400(\mathrm{Z}=5400)$. As evident, the solution was optimum and a feasible region is also obtained using 'NumPy' and 'Matplotlib' library in python.

The true potential of optimization is showcased when we try to solve real world complex problem. As the problem gets complex, it makes sense to move from a manual to more say, efficient and automated, mode of solution hunting process. That's when we can leverage python.

\section{REFERENCES}

[1] Linear Programming by George B. Dantzig.

[2] Optimization with PuLP- PuLP 1.6.0 documentation https://pythonhosted.org/PuLP/

[3] Introduction guide on Linear Programming for (aspiring) data scientists. https://www.analyticsvidhya.com/blog/2017/02

[4] Numerical \& Scientific Computing with Python: Introduction into NumPy https://www.python-course.eu/numpy.php

[5] Matplotlib: Python plotting - Matplotlib 3.0.0 documentation

[6] https://matplotlib.org/ 